\title{
INEQUALITIES FOR THE DIFFERENCE BODY OF A CONVEX BODY
}

\section{G. D. CHAKERIAN}

1. Introduction. Let $K$ be a convex body, i.e. a compact convex set having interior points, in $n$-dimensional Euclidean space $E_{n}$. The "difference body" of $K$, denoted by $D K$, is the centrally symmetric convex body (centered at the origin) defined by,

$$
D K=K+(-K)=\{x-y: x \in K, y \in K\} .
$$

It is well known that $D K$ can equivalently be described as follows,

$$
D K=\{x:(x+K) \cap K \neq \varnothing\} .
$$

In the following, $S$ will denote the boundary of the unit ball in $E_{n}$, and $u$ a variable point of $S$ (so $u$ is a unit vector, or "direction"). The polar equation of the boundary of $D K$ is given by $\rho=\rho(u), u \in S$, so $\rho(u)$ is the radius of $D K$ in the direction $u$. Then $\rho(u)$ is the maximum length of a chord of $K$ having direction $u$-the length of a "diameter" of $K$ having direction $u$.

Let $\mu$ denote $n$-dimensional Lebesque measure in $E_{n}$. Then Rogers and Shephard [5] proved that

$$
\mu(D K) \leqq\left(\begin{array}{c}
2 n \\
n
\end{array}\right) \mu(K),
$$

where equality holds if and only if $K$ is a simplex. They later gave a more geometrical proof in [6]. In $\$ 2$ we give a proof of (3) which clarifies its relationship with the Brunn-Minkowski Theorem and leads to the following generalization, proved at the end of $\S 3$. Let $\alpha \geqq 1-n$, and let $B(p, q)$ be the Beta function, defined by

$$
B(p, q)=\int_{0}^{1} t^{p-1}(1-t)^{q-1} d t
$$

Then

$$
\int_{K \times K}|x-y|^{\alpha} d \mu(x) d \mu(y) \geqq B(n+\alpha, n+1) \mu(K) \int_{S} \rho^{n+\alpha}(u) d u,
$$

where $|x-y|$ is the distance, in $E_{n}$, between $x$ and $y$. Equality holds in (5) if and only if $K$ is a simplex. One sees that (3) follows from (5) by setting $\alpha=0$.

Received by the editors October 2, 1965. 
2. The proof of (3) given in [5] depended on a lemma of T. Bang $[1$, Lemma I]. One can derive that lemma from the following, proved by Fáry and Rédei [4].

LEMMA 1. Let $K_{1}$ and $K_{2}$ be convex bodies in $E_{n}$. Let $\mathcal{F}$ be the family of convex sets $\left(x+K_{1}\right) \cap K_{2}$, with $x$ ranging over $B=\left\{x:\left(x+K_{1}\right) \cap K_{2} \neq \varnothing\right\}$. Then $\mathcal{F}$ is a concave family.

The Brunn-Minkowski Theorem then implies the following lemma, which is simply a restatement of $[4$, Satz 3$]$.

Lemma 2. Let $K_{1}$ and $K_{2}$ be convex bodies in $E_{n}$, and let $f$ be the function defined by $f(x)=\mu\left(\left(x+K_{1}\right) \cap K_{2}\right)^{1 / n}$, with domain $B=\left\{x:\left(x+K_{1}\right) \cap K_{2} \neq \varnothing\right\}$. Then $f$ is a concave function on $B$.

The following proof of (3) is only a slight variant of the first proof of Rogers and Shephard.

Proof of (3). Let $(r, u)$ be spherical coordinates for $E_{n}$, so $r$ represents distance from 0 and $u$ ranges over the unit sphere $S$ centered at 0 . Let $f(x)=\mu((x+K) \cap K)^{1 / n}$, for $x \in D K$. Then $f$ is concave, by Lemma 2, and $(f(0))^{n}=\mu(K)$. Let $g(x)=f(0)(1-r / \rho), x \in D K$, where $r=|x|$ is the distance from $x$ to 0 , and $\rho=\rho(x / r)$ is the radius of $D K$ in the direction of $x$. Then $g$ vanishes on the boundary of $D K$, $g(0)=f(0)$, and $g$ is linear on each segment joining 0 to the boundary of $D K$ (the ordinate set of $g$ is a cone in $E_{n+1}$ with altitude $f(0)$, base $D K$, and is contained in the ordinate set of $f$ ). The concavity of $f$ implies $f(x) \geqq g(x), x \in D K$. Thus

(6)

$$
\begin{aligned}
\int_{D K} \mu((x+K) & \cap K) d \mu(x)=\int_{D K} f^{n} d \mu \geqq \int_{D K} g^{n} d \mu \\
= & (f(0))^{n} \int_{S} \int_{0}^{\rho}(1-r / \rho)^{n} r^{n-1} d r d u \\
& =\mu(K) \int_{S} \rho^{n} \int_{0}^{1} t^{n-1}(1-t)^{n} d t d u \\
& =\left(\begin{array}{c}
2 n \\
n
\end{array}\right)^{-1} \mu(K) \frac{1}{n} \int_{S} \rho^{n} d u=\left(\begin{array}{c}
2 n \\
n
\end{array}\right)^{-1} \mu(K) \mu(D K) .
\end{aligned}
$$

With the following easy identity (see $[5,(7)]$ ),

$$
\mu(K)^{2}=\int_{D K} \mu((x+K) \cap K) d \mu(x),
$$

the inequality (3) follows. Equality holds in (3) if and only if equality holds throughout in (6), so that $f=g$. Then $f$ is linear on each segment 
joining 0 to the boundary of $D K$. By the Brunn-Minkowski Theorem, this happens if and only if $(x+K) \cap K$ is positively homothetic to $K=(0+K) \cap K$ whenever $(x+K) \cap K$ is nondegenerate. It is proved in [5] that this property characterizes simplices; hence equality holds in (3) if and only if $K$ is a simplex. (The proof of this characterization of simplices is simplified in [3].) This completes the proof.

The idea used in the above proof suggests the following theorem, whose proof is omitted since it follows precisely the same lines as above.

THEOREM 1. Let $f$ be a nonnegative concave function defined on a convex body $B$ in $E_{n}$. Let $x_{0} \in B$, and let $h(\xi), \xi \geqq 0$, be a strictly increasing real-valued function of a real variable. Then

$$
\int_{B} h(f(x)) d \mu(x) \geqq n \mu(B) \int_{0}^{1} h\left(t f\left(x_{0}\right)\right)(1-t)^{n-1} d t,
$$

where equality holds if and only if $f$ vanishes on the boundary of $B$ and is linear on each segment joining $x_{0}$ to the boundary of $B$.

Note that (3) follows from (8) by taking $B=D K, x_{0}=0, f(x)$ $=\mu((x+K) \cap K)^{1 / n}, h(\xi)=\xi^{n}$.

The following theorem, which was used in [6] in order to prove (3), follows immediately from Theorem 1.

TheOREM 2. Let $K$ be an $(r+s)$-dimensional convex body. Let $R$ be an $r$-dimensional section of $K$ with $r$-measure $\mu(R)$, and let $S$ be the projection of $K$ onto an $s$-dimensional subspace totally orthogonal to $R$. Let $\mu(S)$ be the s-measure of $S$. Then

$$
\left(\begin{array}{c}
r+s \\
s
\end{array}\right) \mu(K) \geqq \mu(R) \mu(S),
$$

where equality holds if and only if each r-dimensional section of $K$ parallel to $R$ is positively homothetic to $R$.

Proof. In Theorem 1, take $n=s, B=S, h(\xi)=\xi r, f(x)=\mu(R(x))^{1 / r}$, for $x \in S$, where $R(x)$ is the $r$-dimensional section of $K$ by the $r$-flat orthogonal to $S$ passing through $x$, and $\mu(R(x))$ is the $r$-measure of $R(x)$. Let $R\left(x_{0}\right)=R$. By the Brunn-Minkowski Theorem, $f$ is concave on $S$. Hence, from (8), we have,

$$
\begin{aligned}
\mu(K) & =\int_{S} \mu(R(x)) d \mu(x) \geqq s \mu(S) \mu(R) \int_{0}^{1} t^{r}(1-t)^{s-1} d t \\
& =\mu(S) \mu(R)\left(\begin{array}{c}
r+s \\
s
\end{array}\right)^{-1} .
\end{aligned}
$$


Equality holds if and only if $f$ is linear on each segment joining $x_{0}$ to the boundary of $S$. This is the case if and only if all the $R(x)$ are homothetic. This completes the proof.

3. The position of an oriented line $G$ in $E_{n}$ can be fixed by specifying its direction $u$ and the point $p$ where $G$ intersects the orthogonal hyperplane through the origin. The "integral-geometric density" $d G$, for oriented lines is then given by

$$
d G=d p d u,
$$

where $d p$ is the $(n-1)$-dimensional volume element in the orthogonal hyperplane, and $d u$ is the element of surface area of the unit sphere $S$. If $K$ is a convex body in $E_{n}$, let $\sigma(G)=\sigma(p, u)$ be the length of the chord $K \cap G$, for each oriented line $G=G(p, u)$, where $p$ and $u$ are as described above. Let

$$
I_{\alpha}(K)=\int \sigma^{\alpha}(G) d G=\int_{S} \int_{K_{u}} \sigma^{\alpha} d p d u,
$$

where $K_{u}$ is the orthogonal projection of $K$ onto a hyperplane orthogonal to $u$, and $d p$ is the volume element in that hyperplane.

TheOREM 3. Let $K$ be a convex body in $E_{n}$, and let $\rho=\rho(u), u \in S$, be the polar equation of $D K$. Then if $m$ is a real number $\geqq 1$, we have

$$
I_{m+1}(K) \geqq m(m+1) B(m, n+1) \mu(K) \int_{S} \rho^{m}(u) d u,
$$

where equality holds if and only if $K$ is a simplex.

Proof. The concavity of $\mu((x+K) \cap K)^{1 / n}$ implies (see the proof of $(3)$ in $\$ 2)$

$$
\mu((x+K) \cap K) \geqq \mu(K)(1-r / \rho)^{n} ;
$$

with $r=|x|$, and $\rho=\rho(x / r)=\rho(u), x \in D K$. Multiply both sides of (14) by $r^{m-1}$ and integrate with respect to $r$, to obtain

$$
\begin{aligned}
\int_{0}^{\rho} r^{m-1} \mu((x+K) \cap K) d r & \geqq \mu(K) \int_{0}^{\rho} r^{m-1}(1-r / \rho)^{n} d r \\
& =\mu(K) B(m, n+1) \rho^{m}(u) .
\end{aligned}
$$

Let $l(p, u)$ be the length of the intersection of the line $G(p, u)$ with $(x+K) \cap K$, where $u=x /|x|$. Then $l(p, u)=\sigma(p, u)-r$, when $\sigma \geqq r$, and 


$$
\begin{aligned}
\int_{0}^{\rho} r^{m-1} \mu((x+K) \cap K) d r & =\int_{0}^{\rho} r^{m-1} \int_{K_{u}} l(p, u) d p d r \\
& =\int_{K_{u}} \int_{0}^{\rho} r^{m-1} l(p, u) d r d p \\
& =\int_{K_{u}} \int_{0}^{\sigma} r^{m-1}(\sigma-r) d r d p \\
& =\frac{1}{m(m+1)} \int_{K_{u}} \sigma^{m+1}(p, u) d p
\end{aligned}
$$

Then (15) and (16) yield

$$
\frac{1}{m(m+1)} \int_{K_{u}} \sigma^{m+1}(p, u) d p \geqq \mu(K) B(m, n+1) \rho^{m}(u) .
$$

Integration of (17) with respect to $u$ yields (13). Equality holds in (13) if and only if it holds in (14), for all $x \in D K$. It follows (as in the proof of (3) in §2) that this happens if and only if $K$ is a simplex. This completes the proof.

In (16), set $m=n$ and integrate both sides over $S$. This gives

$$
\int_{S} \int_{0}^{\rho} \mu((x+K) \cap K) r^{n-1} d r d u=\frac{1}{n(n+1)} I_{n+1}(K) .
$$

Using (7), we then have

$$
I_{n+1}(K)=n(n+1) \mu(K)^{2} .
$$

This is a generalization to $E_{n}$ of an integral-geometric formula of Crofton (see $[2$, p. 20]). If we set $m=n$ in (13) and use (19), we obtain the Rogers-Shephard inequality (3).

In order to prove (5), we need a generalization of the formula of Crofton given in [2, p. 19]. The integral-geometric density for pairs of points $x, y \in E_{n}$ is $d \mu(x) d \mu(y)$. If $G=G(p, u)$ is the line through $x$ and $y$, then a straightforward computation shows that

$$
d \mu(x) d \mu(y)=|x-y|^{n-1} d t_{1} d t_{2} d p d u,
$$

where $|x-y|$ is the distance from $x$ to $y, t_{1}$ is the distance of $x$ from a fixed point of $G$, and $t_{2}$ is the distance of $y$ from a fixed point of $G$. Multiplying both sides of (20) by $|x-y|^{\alpha}, \alpha \geqq 1-n$, and integrating over all pairs of points $x, y \in K$ yields 
(21) $\int_{K \times K}|x-y|^{\alpha} d \mu(x) d \mu(y)=\frac{1}{(n+\alpha)(n+\alpha+1)} I_{n+\alpha+1}(K)$.

If we set $m=n+\alpha$ in (13), and apply (21), we obtain (5).

\section{REFERENCES}

1. Thфger Bang, On covering by parallel-strips, Mat. Tidsskrift. B (1950), 49-53.

2. W. Blaschke, Vorlesungen über Integral-geometrie, reprint, New York, 1949.

3. H. G. Eggleston, B. Grünbaum and V. Klee, Some semicontinuity theorems for convex polytopes and cell-complexes, Comment. Math. Helv. 39 (1964), 165-188.

4. I. Fáry and L. Rédei, Der zentralsymmetrische Kern und die zentralsymmetrische Hïlle von konvexen Körpern, Math. Ann. 122 (1950), 205-220.

5. C. A. Rogers and G. C. Shephard, The difference body of a convex body, Arch. Math. 8 (1957), 220-233.

6. - Convex bodies associated with a given convex body, J. London Math. Soc. 33 (1958), 270-281.

University of California, Davis 